\title{
Tailoring of electromagnetic field localizations by two- dimensional graphene nanostructures
}

\author{
Ze-Bo Zheng ${ }^{1,2}$, Jun-Tao Li ${ }^{2}$, Teng $\mathrm{Ma}^{3}$, Han-Lin Fang ${ }^{2}$, Wen-Cai Ren ${ }^{3}$, Jun Chen ${ }^{1}$, Jun-Cong She ${ }^{1}$, Yu Zhang ${ }^{1}$, \\ Fei Liu ${ }^{1}$, Huan-Jun Chen ${ }^{1}$, Shao-Zhi Deng ${ }^{1}$ and Ning-Sheng $\mathrm{Xu}^{1}$
}

Graphene has great potential for enhancing light-matter interactions in a two-dimensional regime due to surface plasmons with low loss and strong light confinement. Further utilization of graphene in nanophotonics relies on the precise control of light localization properties. Here, we demonstrate the tailoring of electromagnetic field localizations in the mid-infrared region by precisely shaping the graphene into nanostructures with different geometries. We generalize the phenomenological cavity model and employ nanoimaging techniques to quantitatively calculate and experimentally visualize the two-dimensional electromagnetic field distributions within the nanostructures, which indicate that the electromagnetic field can be shaped into specific patterns depending on the shapes and sizes of the nanostructures. Furthermore, we show that the light localization performance can be further improved by reducing the sizes of the nanostructures, where a lateral confinement of $\lambda_{0} / 180$ of the incidence light can be achieved. The electromagnetic field localizations within a nanostructure with a specific geometry can also be modulated by chemical doping. Our strategies can, in principle, be generalized to other two-dimensional materials, therefore providing new degrees of freedom for designing nanophotonic components capable of tailoring two-dimensional light confinement over a broad wavelength range.

Light: Science \& Applications (2017) 6, e17057; doi:10.1038/lsa.2017.57; published online 6 October 2017

Keywords: chemical doping; electromagnetic field localizations; graphene; graphene nanostructures; graphene plasmons

\section{INTRODUCTION}

When the sizes of the devices approach the nanoscale, focusing and manipulating light becomes a great challenge in both scientific research and industrial manufacturing. Nanostructure sustaining surface plasmons (SP) have been demonstrated to be excellent candidates for confining the light field at the nanoscale ${ }^{1,2}$. The most involved plasmonic materials include metal nanostructures and highly doped semiconducting nanostructures, which exhibit SP resonances ranging from ultraviolet to near-infrared regions ${ }^{3-7}$. For remote, non-destructive and highly sensitive detection and imaging, the operation wavelengths of the SP are preferred to be in the mid-infrared or terahertz regions ${ }^{8-10}$. Graphene with atomic thickness has been shown to support strong SP in these regions ${ }^{11-15}$. The collective oscillations of the Dirac fermions can induce strong graphene SP with long lifetimes, which are able to realize extremely spatial confinement of the electromagnetic field in a broad band. These exceptional characteristics have made graphene an outstanding platform for light focusing and manipulation in the two-dimensional regime ${ }^{14,15}$, which can benefit applications in optical communication ${ }^{16,17}$, sensing and detection ${ }^{17-20}$ and imaging $^{21}$.
One of the most intriguing merits of graphene is that the plasmonrelated characteristics are amenable to wide-range tuning by adjusting the charge carrier density, surrounding dielectric environment and the geometries of the graphene flakes ${ }^{12,13,15,22-24}$. In particular, structuring the graphene monolayer into flakes with nanometer sizes can lead to optical near-fields with specific spatial distributions, whereby the electromagnetic field will experience further confinements ${ }^{25-27}$. However, most of the reported graphene nanostructures are either formed adventitiously during mechanical exfoliation or fabricated with limited geometries $^{26-30}$, which limit the tunability of electromagnetic field localizations while preventing elucidation of the relationship between the light field localizations and geometries of the graphene nanostructures. The latter is associated with a more general topic that remains elusive, the electromagnetic field localization behaviors and related mechanisms in two-dimensional nanostructures of atomic thicknesses.

Here, from both the theoretical and experimental perspectives, we explored tailoring of the electromagnetic field localizations in the midinfrared region using monolayers of graphene two-dimensional nanostructures of different shapes and sizes. We generalized the phenomenological cavity model $^{31}$ to visualize the electromagnetic field distributions within the graphene nanostructures. The theoretical

\footnotetext{
${ }^{1}$ State Key Laboratory of Optoelectronic Materials and Technologies, Guangdong Province Key Laboratory of Display Material and Technology, School of Electronics and Information Technology, Guangzhou 510275, China; ${ }^{2}$ State Key Laboratory of Optoelectronic Materials and Technologies, School of Physics, Sun Yat-sen University, Guangzhou 510275, China and ${ }^{3}$ Shenyang National Laboratory for Materials Science, Institute of Metal Research, Chinese Academy of Sciences, Shenyang 110016, China Correspondence: HJ Chen, Email: chenhj8@mail.sysu.edu.cn; SZ Deng, Email: stsdsz@mail.sysu.edu.cn; NS Xu, Email: stsxns@mail.sysu.edu.cn Received 14 November 2016; revised 24 March 2017; accepted 28 March 2017; accepted article preview online 31 March 2017
} 
predictions were corroborated by mapping the electromagnetic field distributions within the various graphene nanostructures using a nanoimaging technique. We further demonstrated that the localizations of the light field could be modulated with chemical doping of the graphene nanostructures. Our results can provide guidelines for the design of graphene nanostructures with superior light focusing capabilities, and help elucidate the SP mechanisms and therewith the dynamics of the Dirac fermions in the graphene nanostructures.

\section{MATERIALS AND METHODS}

\section{Fabrication of graphene nanostructures}

Single-crystalline monolayer graphene was grown on $180-\mu \mathrm{m}$ thick Pt foils ( 99.9 wt $\%$ metal basis, $10 \times 20 \mathrm{~mm}$ ) using the ambientpressure chemical vapor deposition method ${ }^{32}$. The flow rate of the reaction gas and reaction temperature were finely controlled to obtain single-crystalline graphene flakes with a domain size of $\sim 400 \mu \mathrm{m}$. The graphene was transferred onto a silicon substrate with a 290 -nm oxide layer using the electrochemical bubbling transfer method ${ }^{33}$. Thereafter, graphene nanostructures of different shapes and sizes were fabricated using high-resolution electron beam lithography combined with soft $\mathrm{O}_{2}$-ion etching. The plasma etching duration was $1.5 \mathrm{~min}$. The polymethyl methacrylate (PMMA) resist was removed in acetone, which was followed by annealing the graphene samples in an $\mathrm{Ar} / \mathrm{H}_{2}$ atmosphere $(150 / 350 \mathrm{sccm})$ at $450{ }^{\circ} \mathrm{C}$ for $2 \mathrm{~h}$ to remove any organic residues.

\section{Chemical doping of the graphene nanostructures}

Chemical doping was conducted by exposing the graphene nanostructures to $\mathrm{HNO}_{3}$ vapor (68\% in volume). The sample was positioned so that the graphene was exposed to the acid vapor. The separation between the $\mathrm{HNO}_{3}$ solution and sample surface was $\sim 5 \mathrm{~cm}$. The doping process lasted for more than $12 \mathrm{~h}$ to ensure the uniform adsorption of $\mathrm{HNO}_{3}$ onto the graphene surface.

\section{Nanoimaging of the graphene nanostructures}

Nanoimaging was conducted using a scattering-type near-field optical microscope (NeaSNOM, Neaspec GmbH, Munich, Germany). In a specific measurement, a metal-coated atomic force microscope (AFM) tip (Arrow-IrPt, Nanoworld, Neuchâtel, Switzerland) was illuminated using a mid-infrared laser (Access Laser, Everett, WA, USA) with a wavelength range of $9.20-10.70 \mu \mathrm{m}$. The tip was vibrated vertically with a frequency of $\sim 280 \mathrm{kHz}$. The backscattered light from the tip was detected in a pseudoheterodyne interferometric manner, where the scattered light was demodulated at the fourth harmonic of the tip vibration frequency. The optical and morphological images of the sample can be simultaneously obtained by scanning the sample below the tip.

\section{RESULTS AND DISCUSSION}

\section{Tailoring the localization of the electromagnetic fields by the graphene nanostructures: principles}

The principles for localizing the electromagnetic fields in the graphene nanostructures are schematically illustrated in Figure 1. The free-space light field is first coupled onto the graphene via excitation of the SP using the phase matching technique (e.g., a dipole source, a metalized tip or grating structures). Thereafter, the SP waves propagate within the graphene until they encounter a boundary where strong reflection of the SP waves occurs. Then, the reflected and incidence light-launched waves will interfere with each other to form standing wave stripes (Figure 1a). This scenario has been commonly reported in semi-infinite graphene flakes and graphene ribbons ${ }^{12,13,26}$. Alternatively, more SP reflectors can be generated by intentionally increasing the number of boundaries to form nanostructures, whereby back-and-forth reflections of the SP waves in different directions can be realized and complex interference patterns can form (Figure 1b-1g). Furthermore, the interference patterns can be tuned by adjusting the propagation lengths of the SP waves by controlling the sizes of the nanostructures with fixed shapes. In these regards, one can tailor the localizations of the electromagnetic field by precisely modifying the geometries of the graphene nanostructures.

To verify the aforementioned principles, we performed theoretical calculations of the SP interference patterns in graphene nanostructures with different boundaries. The calculations were based on a phenomenological cavity model ${ }^{31}$ that was generalized in our study to consider multiple boundaries in the nanostructures. The graphene SP waves were launched by a metal tip from an AFM (Figure 2a). The plasmon waves within the graphene nanostructures were the sum of the tiplaunched plasmon wave and those reflected from the boundaries (Supplementary Fig. S1),

$$
\psi=\tilde{\psi}_{\mathrm{sp}, 0}+\sum_{j} \tilde{\psi}_{\mathrm{sp}, j}
$$

where $\tilde{\psi}_{\mathrm{sp}, 0}$ is the tip-launched plasmon wave, and the waves reflected by the boundaries can be described as $\tilde{\psi}_{\mathrm{sp}, j}=R_{j} \times \tilde{\psi}_{\mathrm{sp}, 0} \exp \left\{-\left(\frac{4 \pi}{\lambda_{\mathrm{sp}}}\right) r_{j}\left(\gamma_{\mathrm{sp}}+i\right)\right\}$. Parameters $R_{j}, \gamma_{\mathrm{sp}}$ and $r_{j}$ describe the reflection coefficient, plasmon damping rate and distance between the graphene edge and AFM tip, respectively. The interference patterns are associated with $|\psi|$. To simplify the analysis, in the calculations, we fixed $\tilde{\psi}_{\mathrm{sp}, 0}, \lambda_{\mathrm{sp}}$ and $\gamma_{\mathrm{sp}}$ as $1,200 \mathrm{~nm}$ and 0.205 , respectively. The plasmon wavelength of $200 \mathrm{~nm}$ corresponds to a Fermi energy $\left(E_{\mathrm{F}}\right)$ of $0.405 \mathrm{eV}$. One should note that choosing different values for these parameters only modifies the fine details of the results, whereas the underlying physics will not be affected.

The calculated result for a 600 -nm wide $\left(3 \lambda_{\mathrm{sp}}\right)$ nanoribbon was given in Figure $2 b$, which exhibited two bright fringes close to the boundaries. In addition, more fringes with much weaker intensities can be observed in the interior part. The separation between adjacent fringes was $\sim 0.5 \lambda_{\mathrm{sp}}$. The appearance of these bright fringes was the result of constructive interference of the SP waves, whereas deterioration of the inner fringes was due to damping of the reflected plasmon waves by the graphene (Figure 1a). These observations were consistent with previous experimental studies in graphene nanoribbons ${ }^{12,13,26}$. We then increased the number of boundaries to form more complex graphene nanostructures and calculated the corresponding SP interferences (Figure $2 \mathrm{c}-2 \mathrm{~g}$ ). For the polygonal nanostructures, the separations between the cavity centers and boundaries were maintained at $300 \mathrm{~nm}\left(\sim 1.5 \lambda_{\mathrm{sp}}\right)$. This value was also used for the radius of the circular nanostructure. Several characteristics can be summarized from the interference patterns of the various nanostructures. First, the SP waves can experience multi-beam interferences due to additional reflections from the boundaries, giving rise to a variety of bright and dark features that were strongly dependent on the geometries of the nanostructures. Most interestingly, the distributions of these features followed similar symmetries of the nanostructures. For example, in the nanostructure of the equilateral-triangle shape, the bright (dark) spots resulting from constructive (destructive) interferences exhibited a $\mathrm{D}_{3^{-}}$ group symmetry (Figure 2c). For the other structures of square, regular pentagon and regular hexagon shapes, their interference patterns were determined by the specific symmetry groups of the nanostructures $\left(D_{4^{-}}, D_{5^{-}}\right.$and $D_{6^{-}}$groups, respectively, in Figure $2 \mathrm{~d}-$ $2 \mathrm{f})$. The interference of the circular nanostructure generated ring- 
shaped patterns with the highest symmetry of the $D_{\infty}$-group (Figure $2 \mathrm{~g}$ ). These observations strongly suggest that by multiple reflections, the graphene nanostructures can pattern the tip-launched SP waves of centrosymmetry into complex localized fields with nearfield distributions corresponding to the specific nanostructure geometries.

Second, the interference intensities were strongest next to the boundaries and degraded away from the edges. This phenomenon is due to plasmon damping, as mentioned before. In addition, the field localizations were strongest at the regions close to the corner sites in the nanostructures with sharp corners (Figure 2c-2f). This finding suggests that the localized electromagnetic fields can be further enhanced with nanostructures of small boundary separations, which will be discussed in detail below. The third interesting observation is the so-called edge plasmon modes manifested by bright and dark spots decorating the boundaries (Figure $2 \mathrm{c}-2 \mathrm{f}$ ). In previous studies, the appearance of these edge modes was ascribed to resonances induced by interference between the tip-launched SP waves and those reflected by two corners connecting the boundary ${ }^{27}$ or between SP waves reflected by graphene defects distributed along the boundary ${ }^{26}$. Because we did not take any defect sites into account in our calculations, the observed edge plasmon modes in the graphene nanostructures could be attributed to the former origin. However, in previous reports ${ }^{26,27}$, the plasmon wavelengths of the edge modes are slightly shorter than that of the sheet mode, which is due to the reduction of Drude weight at the edge with lower free-carrier concentrations. In our calculation, we assume that the carrier density of the whole graphene sheet is uniform. As a result, the edge mode has the same plasmon wavelength as that of the sheet mode. We should emphasize that the aim of our current study is to demonstrate the dependence of the plasmon interference within the graphene flake on the geometries of the nanostructures. We therefore neglect the edge plasmon modes in both the theoretical and experimental studies.

Tailoring the localization of the electromagnetic fields by the graphene nanostructures: nanoimaging

The nanoimaging technique was utilized to verify the above calculation results. To that end, a set of graphene nanostructures with different geometries was fabricated using microfabrication procedures (Supplementary Fig. S2). Nanoimaging was conducted by illuminating a metal-coated AFM tip ( $\sim 25 \mathrm{~nm}$ in radius) using focused midinfrared light with a wavelength of $10.70 \mu \mathrm{m}$ (Figure 2a). Backgroundfree near-field optical amplitude, $s_{4}$, can be obtained by demodulating the scattered light induced by the interaction between the tip and nanostructures at the fourth harmonic of the tip vibration frequency ${ }^{34,35}$. In such a manner, the localization patterns of the incidence field within the nanostructures can be acquired by recording the real-space near-field images $\left|E_{\mathrm{z}}\right|$ while scanning the nanostructures a

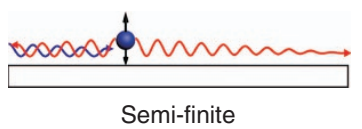

Semi-finite

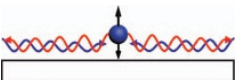

1D cavity b

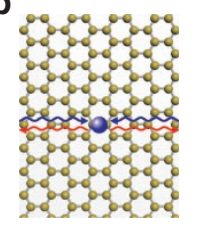

e

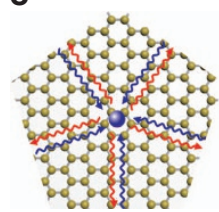

C

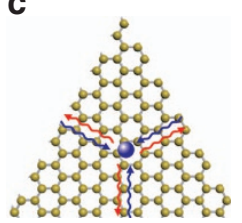

f

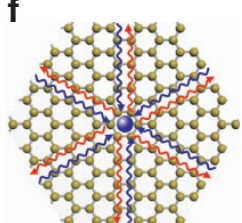

d

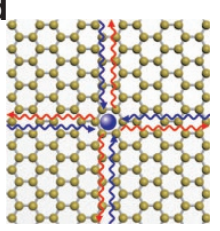

g

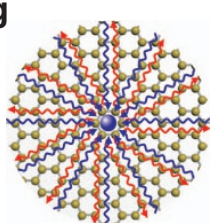

Figure 1 Schematic showing the principles of tailoring the localizations of the electromagnetic field using two-dimensional graphene nanostructures. (a) Sketches of the reflection and interference of the graphene SP waves in the nanostructures with one and two boundaries. (b-f) Sketches of the reflections and interferences of the graphene SP waves within the graphene nanostructures of two, three, four, five and six boundaries. (g) Sketch of the reflection and interference of the graphene SP waves within a circular nanostructure, where the number of the boundaries can be observed as infinite.
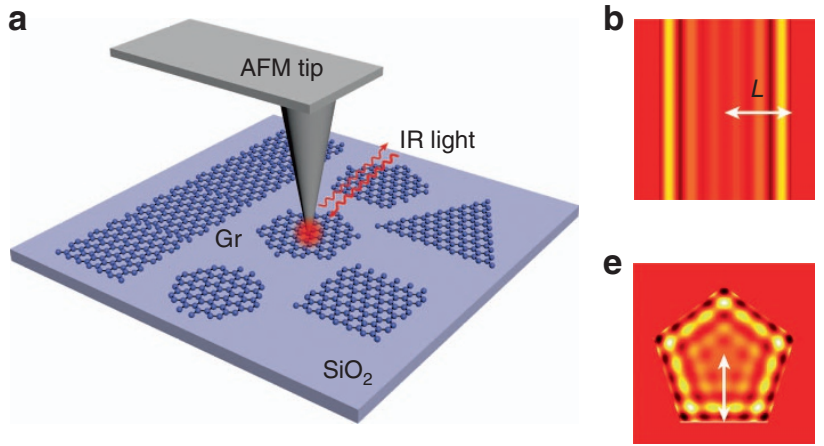

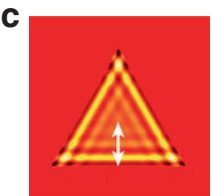

$$
L=1.5 \lambda_{\mathrm{sp}}
$$

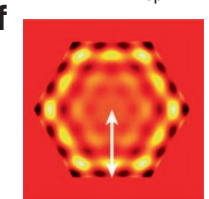

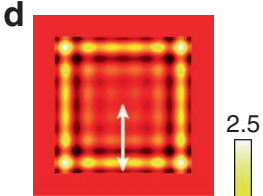

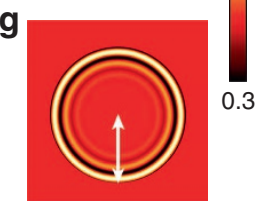

Figure 2 Theoretical calculations of SP interference patterns in the two-dimensional graphene nanostructures. (a) Schematic showing the excitation and

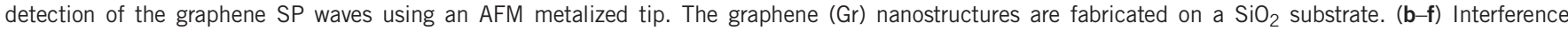
patterns of the graphene SP waves within the graphene nanostructures with two, three, four, five and six boundaries. The white double-headed arrows indicate the separation, $L$, between the centers and boundaries, which are fixed at $1.5 \lambda_{\mathrm{sp}}$. (g) Interference pattern of the graphene SP waves in a circular nanostructure. The white double-headed arrow indicates the radius, which is set as $1.5 \lambda_{\mathrm{sp}}$. 
a

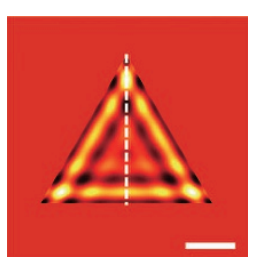

$L=200 \mathrm{~nm}$

f

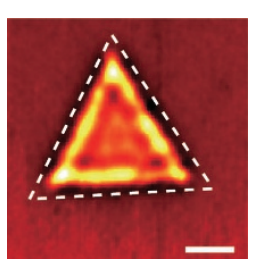

k

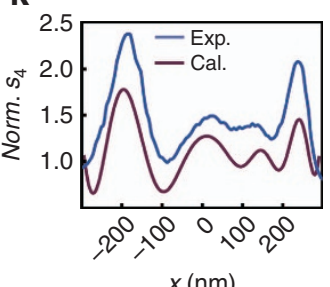

b

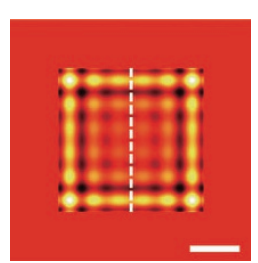

$L=295 \mathrm{~nm}$

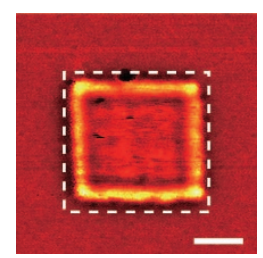

I

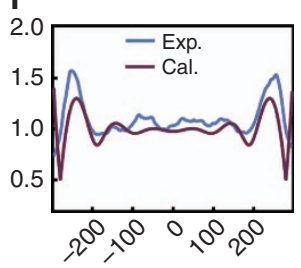

C

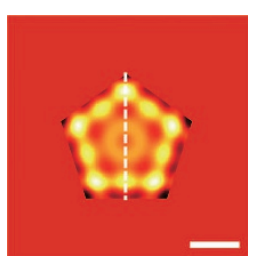

$L=225 \mathrm{~nm}$

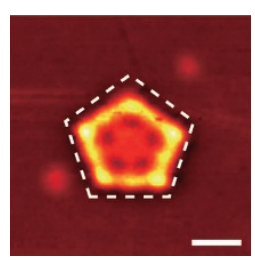

m

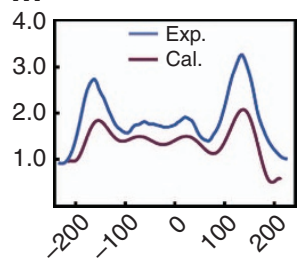

$x(\mathrm{~nm})$ d

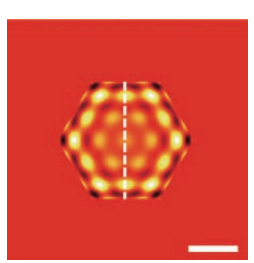

$L=240 \mathrm{~nm}$

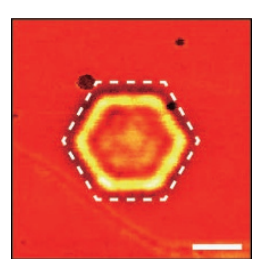

n

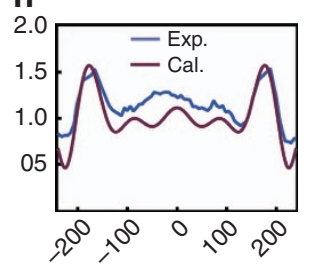

$x(\mathrm{~nm})$ e

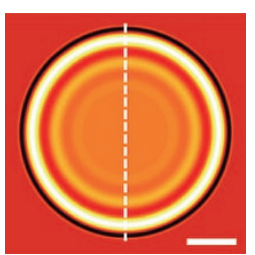

$L=450 \mathrm{~nm}$

j

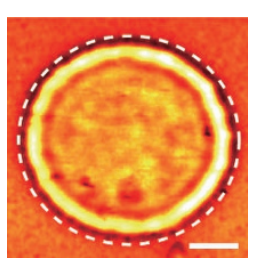

0.3

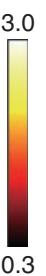

0

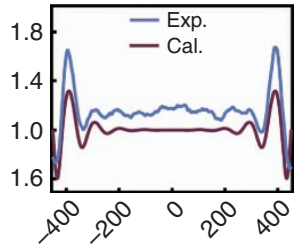

$x(\mathrm{~nm})$

Figure 3 Comparison of the experimental and theoretical results of the SP interference patterns in the two-dimensional graphene nanostructures. (a-e) Theoretical interference patterns of the graphene SP wave within the equilateral triangle, square, regular pentagon, regular hexagon and circular graphene nanostructures. SP wavelengths of $185 \mathrm{~nm}$ are used in the calculations, which correspond to an $E_{\mathrm{F}}$ of $0.375 \mathrm{eV}$. (f-j) Corresponding experimental near-field amplitude images of the graphene nanostructures. The boundaries are indicated by the white dashed lines. (k-o) Extracted experimental (blue) and calculated (red) normalized amplitude profiles along the white dashed lines marked in a-e. The normalizations are performed by dividing the amplitudes of the nanostructures by that of the pristine $\mathrm{SiO}_{2}$ substrate. Scale bars $=200 \mathrm{~nm}$.

under the AFM tip. The measured near-field distributions corroborated most of the interference features predicted (Figure 3a-3j). Specifically, the $\left|E_{\mathrm{z}}\right|$ images of the nanostructures displayed the same symmetries as those calculated. The regions next to the corner sites of each polygonal nanostructure exhibited the strongest localized field amplitudes. In particular, for the regular hexagon nanostructure with an $L$ of $240 \mathrm{~nm}$, the retardation between the center and boundaries was $\sim 3 \lambda_{\mathrm{sp}}$, which resulted in constructive SP interferences at the center and gave well-defined bright spots (Figure $3 \mathrm{~d}$ and $3 \mathrm{i}$ ). For the circular nanostructure, interference rings with attenuated intensities toward the center were observed in the near-field images (Figure $3 \mathrm{e}$ and $3 \mathrm{j}$ ). The agreements between the calculations and nano-images can be observed more clearly by extracting the respective normalized profile $\left|E_{z}\right| /\left|E_{z \text {,sub }}\right|$ along the central axis of each nanostructure (Figure $3 \mathrm{k}-3 \mathrm{o}$ ), where $\left|E_{z \text {,sub }}\right|$ was the near-field amplitude of the pristine substrate adjacent to a specific nanostructure.

In addition to the amplitude imaging, information about the phases was desired for a complete description of the localized electromagnetic fields. To this end, phase-resolved nanoimaging was utilized to measure the spatial phase distributions associated with each interference pattern (Supplementary Fig. S3). Both of the experimental and theoretical phase images exhibited standing wave patterns with spatial symmetries similar to the corresponding amplitude counterparts. In addition, the adjacent bright and dark fringes close to the boundaries differed by $\sim \pi / 2$. These behaviors further confirmed that the localization patterns of the electromagnetic fields were indeed due to constructive and destructive interferences of the SP waves within the graphene nanostructures. To the best of our knowledge, our results are the first images of the phase distributions in the graphene nanostructures of different shapes. Alternatively, we should note that there still remain differences between the experimental and theoretical results, especially for the amplitude and phase contrasts in the inner parts of the nanostructures. We ascribe such discrepancies to the defects and inhomogeneities of the graphene nanostructures incorporated during microfabrication. In addition, the finite size of the AFM tip also precluded high-resolution visualization of the fine near-field features.

Dependence of the localized electromagnetic fields on the sizes of the graphene nanostructures

The size of the nanostructures is another important parameter that can affect the localized electromagnetic fields. To reveal the effects of size, we imaged a series of graphene nanostructures with fixed shapes with various sizes. Their sizes were characterized by the separation $L$ between the centers and boundaries. All these nanostructures exhibited similar evolvements in interference patterns. First, the brightest fringes always existed next to the edges of the nanostructures with different sizes (Figure $4 \mathrm{a}-4 \mathrm{c}, 4 \mathrm{f}-4 \mathrm{~h}$ and $4 \mathrm{k}-4 \mathrm{~m}$ ). For nanostructures with an $L$ smaller than $150 \mathrm{~nm}$, the fringes merged into each other to form a bright center (Figure $4 \mathrm{~d}, 4 \mathrm{i}$ and $4 \mathrm{n}$ ). These behaviors can be further elucidated by the calculated normalized amplitudes along the central axis of each nanostructure, which were consistent with the respective experimental results (Supplementary Figs. S4-S6). Second, bright and dark spots appeared alternately at the centers of the nanostructures with decreasing $L$. These data can be analyzed quantitatively by plotting the near-field signals at each nanostructure center as a function of $L$ (position marked by $a$ in Figure $4 \mathrm{a}, 4 \mathrm{f}$ and $4 \mathrm{k}$ ), which oscillated strongly with decreasing $L$ (Figure $4 \mathrm{e}, 4 \mathrm{j}$ and $4 \mathrm{o}$ ). Such a behavior indicated that at the centers, the constructive and destructive plasmon interferences could both occur, depending on the sizes of the 

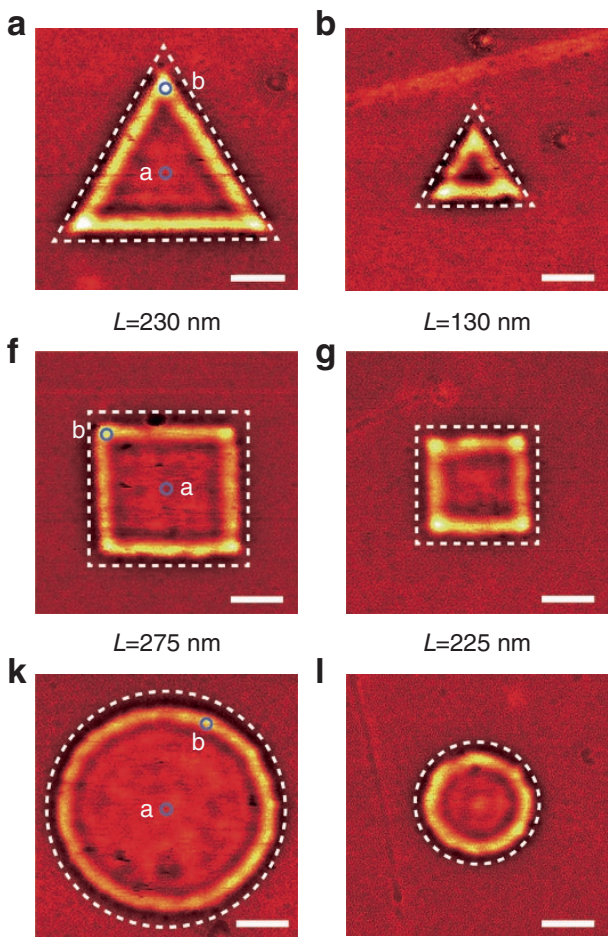

$L=450 \mathrm{~nm}$

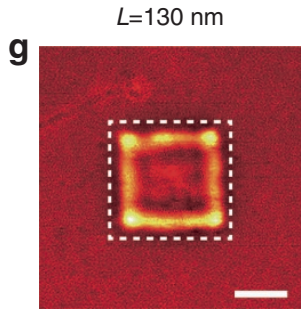

$L=225 \mathrm{~nm}$

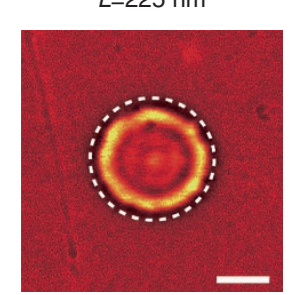

$L=240 \mathrm{~nm}$

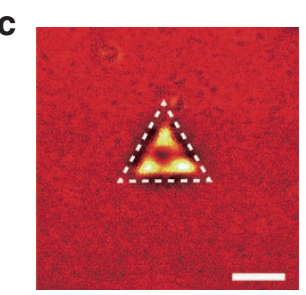

$L=90 \mathrm{~nm}$

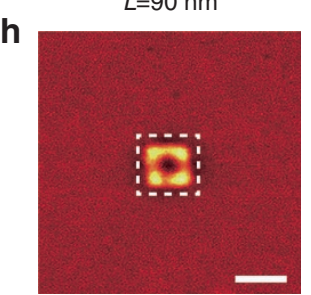

$L=100 \mathrm{~nm}$

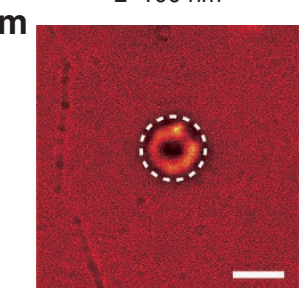

$L=130 \mathrm{~nm}$

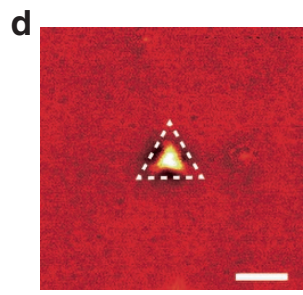

$L=60 \mathrm{~nm}$

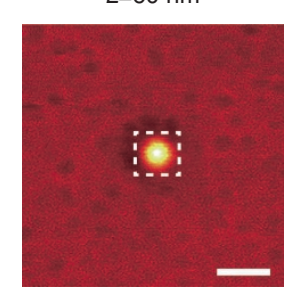

$L=50 \mathrm{~nm}$

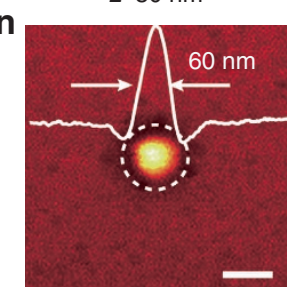

$L=65 \mathrm{~nm}$

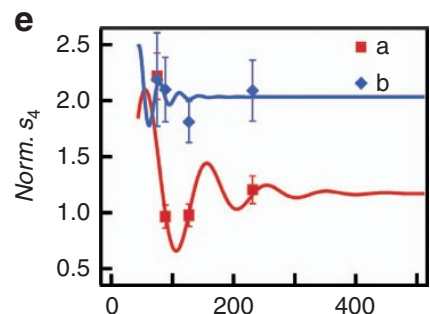

j

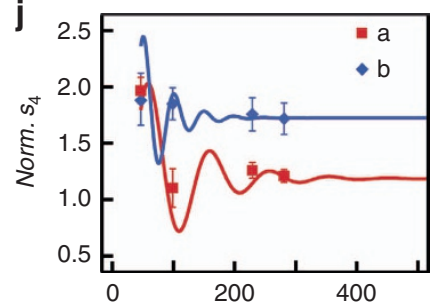

0

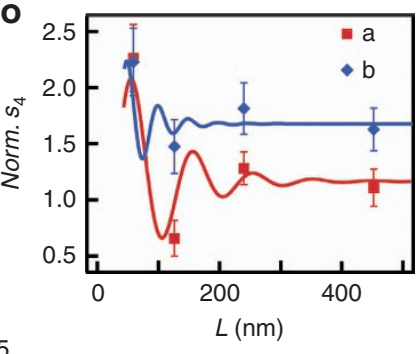

Figure 4 SP interference patterns of the two-dimensional graphene nanostructures with different sizes and shapes. (a-d,f-i,k-n) Near-field amplitude images within the equilateral triangle, square and circular graphene nanostructures of varied sizes. The white dashed lines indicate the boundaries of the graphene nanostructures. (e, j, o) Normalized near-field amplitudes at positions a (red squares) and b (blue diamonds) marked in a, f and k, respectively. The normalizations are performed by dividing the amplitude at a or b by that of the pristine $\mathrm{SiO}_{2}$ substrate. The red and blue solid lines are theoretical results using the phenomenological cavity model. $\mathbf{a}-\mathbf{c}, \mathbf{f}-\mathbf{h}, \mathbf{k}-\mathbf{m}$ Scale bars $=200 \mathrm{~nm}$ and $\mathbf{d}, \mathbf{i}, \mathbf{n}$ Scale bars $=100 \mathrm{~nm}$.

nanostructures. Another interesting behavior was related to the intersections between the two connecting brightest fringes (position marked by $b$ in Figure $4 \mathrm{a}, 4 \mathrm{f}$ and $4 \mathrm{k}$ ). At these positions, the amplitudes were almost invariant until $L$ was smaller than $200 \mathrm{~nm}$, where the influences from the other boundaries became large enough to affect the interference behaviors. These size-dependent characteristics are clear evidence of plasmon resonances in graphene nanostructures ${ }^{27}$.

Alternatively, as the sizes of the nanostructures decreased, the number of the bright spots decreased (Supplementary Figs. S4-S6). Specifically, for nanostructures with $L \sim 50 \mathrm{~nm}$, all the bright features collapsed into bright spots with strong intensities, suggesting a deep subwavelength focusing of the incidence fields. For example, in the circular nanostructure with a diameter of $130 \mathrm{~nm}$, the interference resulted in a bright spot, with a diameter $\sim 60 \mathrm{~nm}$, at the center. The lateral field confinement was down to $\sim 1 / 180$ of the incidence wavelength, which corresponded to a mode volume of $\sim 2.2 \times 10^{-8} \lambda_{0}^{3}$. Such a strong confinement was much larger than that of the traditional metallic plasmonic cavities $\left(10^{-3} \lambda_{0}^{3}\right)$ Ref. 36. The extreme field localizations in the graphene nanostructure could be understood from two aspects. First, the unique electronic structure of the graphene can give mass-less Dirac fermions with ultrahigh mobilities, which results in low-loss plasmon resonances with high electromagnetic field confinements. Second, the field confinements can be further enhanced by the constructive interference of the SPs within the nanostructures. This ultrasmall mode volume can enable various light-matter interactions at subwavelength scale, such as sensing, imaging and photolithography beyond the diffraction limit.

\section{Dependence of localized electromagnetic fields on excitation wavelengths}

To further explore electromagnetic field localizations in graphene nanostructures, we performed nanoimaging on the circular cavity with a diameter of $150 \mathrm{~nm}$ using different excitation wavelengths. As shown in Figure 5a-5e, the intensities of the bright spots at the nanostructure center gradually decreased as the excitation wavelengths decreased. Under an excitation of $9.60 \mu \mathrm{m}$, the localized features could be barely distinguished from the amplitude image. This evolvement can be observed more clearly by plotting the dependence of the central intensities on the excitation wavelengths (Figure 5f). The annihilation of the localized field amplitudes for shorter excitation wavelengths can be ascribed to the damping of the graphene SPs. By reducing the incidence wavelengths, the frequency of the graphene SPs approached the optical phonon absorption of the $\mathrm{SiO}_{2}$ substrate ${ }^{37}$. As a result, the damping of the SPs became stronger, giving rise to weaker near-field amplitudes. This result can be further corroborated by calculating the dependence of $1 / \gamma_{\mathrm{sp}}$ on the incidence wavelengths, which followed the same trend as that of the near-field amplitude at the nanostructure center.

Tailoring the localized electromagnetic fields with chemical doping of the graphene nanostructures

The above studies unambiguously demonstrate that the electromagnetic field localizations can be tailored by tuning the geometries of the graphene nanostructures. Alternatively, the plasmonic properties of the graphene strongly depend on the Fermi energy, which is 

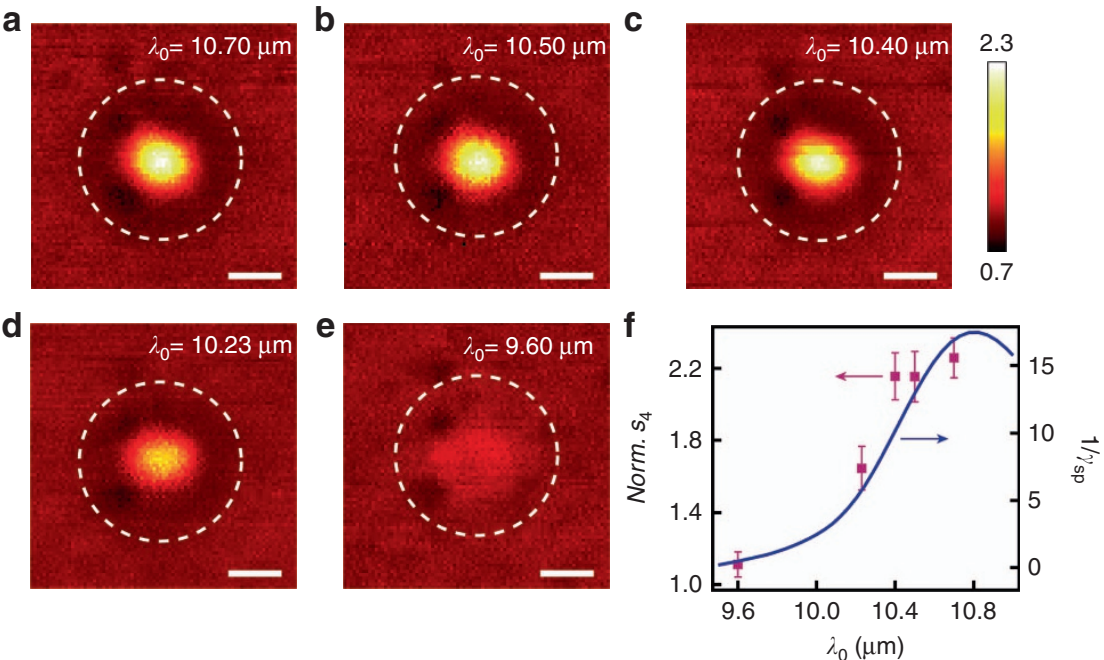

Figure 5 Dependence of SP interference patterns on the excitation wavelengths in a circular graphene nanostructure. (a-e) Near-field amplitude images within the circular graphene nanostructures using different excitation wavelengths. The diameter is $150 \mathrm{~nm}$. Scale bars $=50 \mathrm{~nm}$. The white dashed lines indicate the boundaries. (f) Dependence of the $1 / \gamma_{\mathrm{sp}}$ and near-field amplitudes at the nanostructure center on the excitation wavelengths. The near-field amplitudes are normalized to that of the adjacent pristine $\mathrm{SiO}_{2}$ substrate.

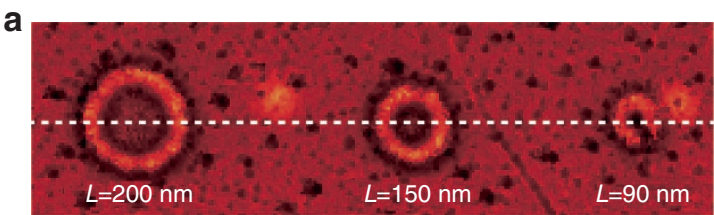

b

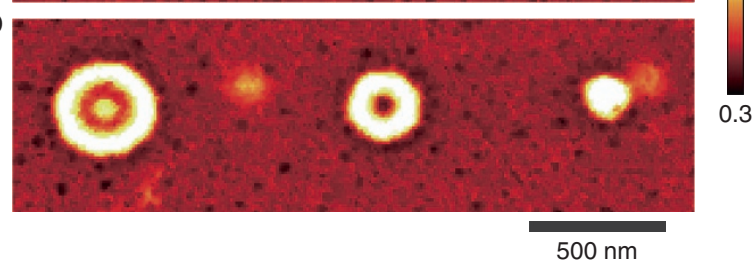

$500 \mathrm{~nm}$

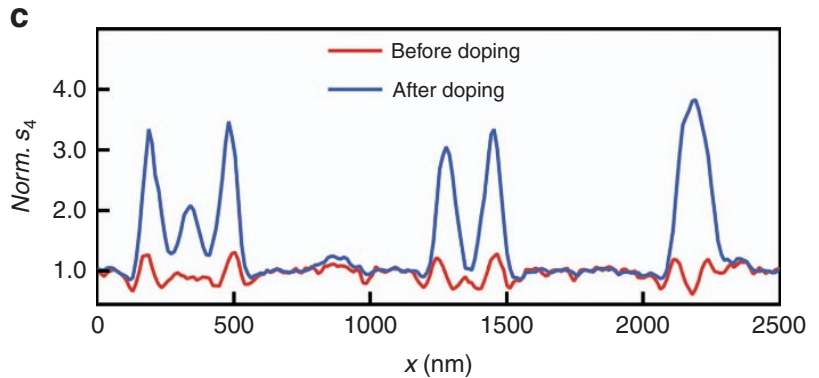

Figure 6 Comparison of near-field optical images of the circular graphene nanostructure before and after $\mathrm{HNO}_{3}$ doping. The excitation wavelength was $\lambda_{0}=10.70 \mu \mathrm{m}$. (a) Normalized near-field optical images of the pristine graphene nanostructures. (b) Normalized near-field optical images of the $\mathrm{HNO}_{3}$-doped graphene nanostructures. (c) Extracted amplitude profiles along the dashed white line which is shown in a. The red and blue solid lines represent the profiles from the pristine and chemically doped graphene nanostructures, respectively.

determined by the free-carrier density as $E_{\mathrm{F}}=h v_{\mathrm{F}} \sqrt{\pi n}$ ( $v_{\mathrm{F}}$ is the Fermi velocity and $n$ is free-carrier density in the graphene) ${ }^{38}$. Therefore, one can manipulate the plasmonics in the graphene by charge carrier-doping approaches, such as electrostatic gating or chemical doping. Our previous study revealed that the graphene plasmon strengths and wavelengths were enhanced by $\mathrm{HNO}_{3}$ doping, which was due to the injection of free holes and the reduction of the plasmon damping rates ${ }^{39}$.

To demonstrate the tunability of the electromagnetic field localizations by chemical doping, the nanostructures of circular shapes with different diameters were exposed to the $\mathrm{HNO}_{3}$ vapor to initiate $p$-type doping. As shown in Figure 6, the electromagnetic fields within the graphene nanodisks were notably modified upon hole injections. In comparison with the pristine samples, the normalized near-filed optical intensities were strongly enhanced by chemical doping (Figure 6a and $6 \mathrm{~b})$. The intensity of the brightest fringe next to the disk edge was strengthened by $\sim 1.64$ times (Figure $6 \mathrm{c}$ ), which is due to the enhancements of the plasmon oscillation strengths with increasing carrier density. In addition, the interference patterns were remarkably modified. Specifically, in the graphene disks with $L=200 \mathrm{~nm}$ and $L=150 \mathrm{~nm}$, the number of bright fringes was reduced, and the fringe separations became larger. For the disk with $L=90 \mathrm{~nm}$, the electromagnetic field was tightly confined to a bright spot (Figure $6 \mathrm{c}$ ). These behaviors originated from the increase of the graphene plasmon wavelengths upon hole injections ${ }^{39}$, whereby the interference nodes within a specific graphene disk are reduced. The dependence of the interference patterns on chemical doping suggests that one can tailor the electromagnetic fields of the graphene nanostructure with chemical doping without modifying the geometry.

The realization of electromagnetic field localization in two dimensions using graphene nanostructures of different geometries can offer several advantages in terms of efficient focusing and manipulating of light at the nanoscale. In the realm of practical applications, our results provide a new degree of freedom for tailoring the graphene plasmonics by fine tuning the shapes and sizes of the graphene nanostructures. These graphene nanostructures can further be integrated with other photonic or optoelectronic devices to enhance performance. Of particular importance is that through fine tuning of the graphene nanostructures, one can pattern the localized electromagnetic fields to determinately overlap with the functional components in these devices, whereby enhancements can be maximized. From the fundamental aspect, the graphene nanostructures can provide a facile platform to cultivate the behaviors of the Dirac electrons and their interactions with light in two dimensions, which can help elucidate the 
quantum nature of plasmonics in the graphene. We also want to emphasize that the strategy of tailoring the localization of electromagnetic field by tuning the geometries of the two-dimensional nanostructures can be generalized to other material systems sustaining surface waves, such as few-layer boron nitrides with surface phonon polaritons and atomically thick two-dimensional semiconductors with exciton polaritons ${ }^{40}$. In such a manner, by choosing the proper materials, one can design novel nanophotonic structures capable of focusing and manipulating electromagnetic fields from the visible to terahertz wavelength ranges. Finally, we would like to note that due to the relatively large size $(\sim 100 \mathrm{~nm})$ of the nanostructures used in our current study, the observed electromagnetic field localization behaviors still follows the predictions from classical electrodynamic theory. However, previous theoretical studies have revealed the exotic quantum behaviors of graphene plasmons in nanostructures with sizes of several nanometers, such as mode splitting, size-dependent plasmon broadening, edge-dependent plasmon broadening and nonlinear optical effects ${ }^{41,42}$. We believe that these quantum phenomena can be explored by fabricating nanostructures with much smaller sizes, which will be carried out in our future studies.

\section{CONCLUSIONS}

In conclusion, we have demonstrated the capability of the graphene two-dimensional nanostructures to tailor the localization of midinfrared electromagnetic fields at the nanoscale. By designing nanostructures of specific shapes, multi-beam interferences between the graphene SP waves can be realized, which lead to intriguing localized electromagnetic field patterns. The regions with strong field enhancements follow symmetries analogous to that of the nanostructures. In addition, constructive and destructive interferences of the SP waves can be modulated by fine tuning the sizes of the nanostructure with specific shapes. In particular, using small nanostructures, the light field can be strongly compressed to a lateral size down to $\sim 1 / 180$ of the incidence wavelength. The electromagnetic field localizations within a nanostructure can also be modulated with chemical doping. We believe that our results can pave the way for the design of graphenebased optical and optoelectronic devices for imaging, detection and sensing in future nanophotonic circuits.

During the preparation of this manuscript, we became aware of another work on a similar topic ${ }^{27}$. Our study was executed independently. In addition, the previous work mainly focused on analyzing the edge and sheet plasmon modes in graphene nanocavities, whereas in our present study, we aimed to demonstrate the new degrees of freedom for tailoring the electromagnetic field localizations in graphene nanostructures by tuning their shapes and sizes.

\section{CONFLICT OF INTEREST}

The authors declare no conflict of interest.

\section{ACKNOWLEDGEMENTS}

We thank Miss SS Li for valuable discussions in sample fabrication. This work was financially supported by the National Natural Science Foundation of China (Grant Nos. 51290271, 11474364 and 51290273), the National Key Basic Research Program of China (Grant Nos. 2013CB933601 and 2013YQ12034506) and the Guangdong Natural Science Funds for Distinguished Young Scholars (Grant No. 2014A030306017). ZZ performed the theoretical calculations and nanoimaging experiments and analyzed the data. HF and JL helped fabricate the graphene nanostructures. TM and WR prepared the single-crystalline monolayer graphene samples. JC, JS, YZ and FL helped discuss the results. HC, SD and NX conceived the study, supervised the project, discussed and interpreted the results, and co-wrote the manuscript.
1 Barnes WL, Dereux A, Ebbesen TW. Surface plasmon subwavelength optics. Nature 2003; 424: 824-830.

2 Schuller JA, Barnard ES, Cai WS, Jun YC, White JS et al. Plasmonics for extreme light concentration and manipulation. Nat Mater 2010; 9: 193-204.

3 Liu N, Guo HC, Fu LW, Kaiser S, Schweizer H et al. Three-dimensional photonic metamaterials at optical frequencies. Nat Mater 2008; 7: 31-37.

4 Qin F, Ding L, Zhang L, Monticone F, Chum CC et al. Hybrid bilayer plasmonic metasurface efficiently manipulates visible light. Sci Adv 2016; 2: e1501168.

5 McClain MJ, Schlather AE, Ringe E, King NS, Liu LF et al. Aluminum nanocrystals. Nano Lett 2015; 15: 2751-2755.

6 Marinica DC, Zapata M, Nordlander P, Kazansky AK, Echenique PM et al. Active quantum plasmonics. Sci Adv 2015; 1: e1501095.

7 Liu N, Mukherjee S, Bao K, Li Y, Brown LV et al. Manipulating magnetic plasmon propagation in metallic nanocluster networks. ACS Nano 2012; 6: 5482-5488.

8 McIntosh AI, Yang B, Goldup SM, Watkinson M, Donnan RS. Terahertz spectroscopy: a powerful new tool for the chemical sciences? Chem Soc Rev 2012; 41: 2072-2082.

9 Jepsen PU, Cooke DG, Koch M. Terahertz spectroscopy and imaging-modern techniques and applications. Laser Photonics Rev 2011; 5: 124-166.

10 Sirtori C, Barbieri S, Colombelli R. Wave engineering with $\mathrm{THz}$ quantum cascade lasers. Nat Photonics 2013; 7: 691-701.

11 Jablan M, Buljan H, Soljačić M. Plasmonics in graphene at infrared frequencies. Phys $\operatorname{Rev} B$ 2009; 80: 245435.

12 Fei Z, Rodin AS, Andreev GO, Bao W, McLeod AS et al. Gate-tuning of graphene plasmons revealed by infrared nano-imaging. Nature 2012; 487: 82-85.

13 Chen JN, Badioli M, Alonso-González P, Thongrattanasiri S, Huth F et al. Optical nanoimaging of gate-tunable graphene plasmons. Nature 2012; 487: 77-81.

14 Koppens FHL, Chang DE, García de Agajo FJ. Graphene plasmonics: a platform for strong light-matter interactions. Nano Lett 2011; 11: 3370-3377.

15 Grigorenko AN, Polini M, Novoselov KS. Graphene plasmonics. Nat Photonics 2012; 6: 749-758.

16 Mueller T, Xia FN, Avouris P. Graphene photodetectors for high-speed optical communications. Nat Photonics 2010; 4: 297-301.

17 Bonaccorso F, Sun Z, Hasan T, Ferrari AC. Graphene photonics and optoelectronics. Nat Photonics 2010; 4: 611-622.

$18 \mathrm{Li}$ YL, Yan HG, Farmer DB, Meng X, Zhu WJ et al. Graphene plasmon enhanced vibrational sensing of surface-adsorbed layers. Nano Lett 2014; 14: 1573-1577.

19 Wang XM, Cheng ZZ, Xu K, Tsang HK, Xu JB. High-responsivity graphene/ silicon-heterostructure waveguide photodetectors. Nat Photonics 2013; 7: 888-891.

20 Koppens FHL, Mueller T, Avouris P, Ferrari AC, Vitiello MS et al. Photodetectors based on graphene, other two-dimensional materials and hybrid systems. Nat Nanotechnol 2014; 9: 780-793.

$21 \mathrm{Li} \mathrm{PN}$, Taubner T. Broadband subwavelength imaging using a tunable graphene-lens. ACS Nano 2012; 6: 10107-10114.

22 Brar VW, Sherrott MC, Jang MS, Kim S, Kim L et al. Electronic modulation of infrared radiation in graphene plasmonic resonators. Nat Commun 2015; 6: 7032.

23 Mikhailov SA, Ziegler K. New electromagnetic mode in graphene. Phys Rev Lett 2007; 99: 016803.

24 Woessner A, Lundeberg MB, Gao YD, Principi A, Alonso-González P et al. Highly confined low-loss plasmons in graphene-boron nitride heterostructures. Nat Mater 2015; 14: 421-425.

25 Thongrattanasiri S, García de Agajo FJ. Optical field enhancement by strong plasmon interaction in graphene nanostructures. Phys Rev Lett 2013; 110: 187401.

26 Fei Z, Goldflam MD, Wu J-S, Dai S, Wagner M et al. Edge and surface plasmons in graphene nanoribbons. Nano Lett 2015; 15: 8271-8276.

27 Nikitin AY, Alonso-González P, Vélez S, Mastel S, Centeno A et al. Real-space mapping of tailored sheet and edge plasmons in graphene nanoresonators. Nat Photonics 2016; 10: 239-243.

28 Jia YC, Zhao H, Guo QS, Wang XM, Wang H et al. Tunable plasmon-phonon polaritons in layered graphene-hexagonal boron nitride heterostructures. ACS Photonics 2015; 2: 907-912.

29 Yang XX, Zhai F, Hu H, Hu DB, Liu RN et al. Far-field spectroscopy and near-field optical imaging of coupled plasmon-phonon polaritons in 2D van der Waals heterostructures. Adv Mater 2016; 28: 2931-2938.

30 Fang ZY, Wang YM, Schather AE, Liu Z, Ajayan PM et al. Active tunable absorption enhancement with graphene nanodisk arrays. Nano Lett 2014; 14: 299-304.

31 Gerber JA, Berweger S, O'Callahan BT, Raschke MB. Phase-resolved surface plasmon interferometry of graphene. Phys Rev Lett 2014; 113: 055502.

$32 \mathrm{Ma} \mathrm{T}$, Ren WC, Liu ZB, Huang L, Ma L-P et al. Repeated growth-etching-regrowth for large-area defect-free single-crystal graphene by chemical vapor deposition. ACS Nano 2014; 8: 12806-12813.

33 Gao LB, Ren WC, Xu HL, Jin L, Wang ZX et al. Repeated growth and bubbling transfer of graphene with millimeter-size single-crystal grains using platinum. Nat Commun 2012; 3. 699 .

34 Stiegler JM, Tena-Zaera R, Idigoras O, Chuvilin A, Hillenbrand R. Correlative infraredelectron nanoscopy reveals the local structure-conductivity relationship in zinc oxide nanowires. Nat Commun 2012; 3: 1131.

35 Chen JN, Nesterov ML, Nikitin AY, Thongrattanasiri S, Alonso-González P et al. Strong plasmon reflection at nanometer-size gap in monolayer graphene on SiC. Nano Lett 2013; 13: 6210-6215. 
36 Russell KJ, Yeung KYM, Hu E. Measuring the mode volume of plasmonic nanocavities using coupled optical emitters. Phys Rev B 2012; 85: 245445.

37 Yan HG, Low T, Zhu WJ, Wu YQ, Freitag M et al. Damping pathways of mid-infrared plasmons in graphene nanostructures. Nat Photonics 2013; 7: 394-399.

38 Castro-Neto AH, Guinea F, Peres NMR, Novoselov KS, Geim AK. The electronic properties of graphene. Rev Mod Phys 2009; 81: 109-162.

39 Zheng ZB, Wang WL, Ma T, Deng SZ, Ke YL et al. Chemically-doped graphene with improved surface plasmon characteristics: an optical near-field study. Nanoscale 2016; 8: $16621-16630$

40 Dai S, Fei Z, Ma Q, Rodin AS, Wagner M et al. Tunable phonon polaritons in atomically thin van der Waals crystals of boron nitride. Science 2014; 343 1125-1129.

41 Thongrattanasiri S, Manjavacas A, García de Agajo FJ. Quantum finite-size effects in graphene plasmons. ACS Nano 2012; 6: 1766-1775.
42 Cox JD, Silveiron I, García de Agajo FJ. Quantum effects in the nonlinear response of graphene plasmons. ACS Nano 2016; 10: 1995-2003.

cc)(i) (2) This work is licensed under a Creative Commons AttributionBY NC SA NonCommercial-ShareAlike 4.0 International License. The images or other third party material in this article are included in the article's Creative Commons license, unless indicated otherwise in the credit line; if the material is not included under the Creative Commons license, users will need to obtain permission from the license

holder to reproduce the material. To view a copy of this license, visit http:// creativecommons.org/licenses/by-nc-sa/4.0/

(C) The Author(s) 2017

Supplementary Information for this article can be found on the Light: Science \& Applications' website (http://www.nature.com/lsa). 